\title{
Multimodal biometric system based on Matching Score Level Fusion of Palm print And Finger print.
}

\author{
${ }^{1}$ Mr.Juberahmad A Shaikh., ${ }^{2}$ Prof Dr.Uttam D.Kolekar \\ Professor and Principal, A.P. Shah Institute of Technology, Thane, Mumbai
}

\begin{abstract}
In multimodal biometric technique strength of unimodal system biometrics are combined to increase recognition accuracy of the system. In this paper palm print and finger print biometrics are used to design robust recognition system. A feature of palm print are extracted with gray level co-occurrence based Harlick features and feature of finger print are extracted with minutiae based techniques. The proposed system is tested on publically available IIT Delhi Touch less Palm print database and FVC 2002 database for finger print. In this paper matching score level fusion technique with weighted sum rule based fusion is applied to fuse their individual score of palm print, finger print traits. The recognition accuracy is improved and it is found good as compared with recognition accuracy of individual traits. The multimodal system is evaluated on the basis of performance parameter, Accuracy $99.93 \%$, and Equal Error Rate (EER) is 0.0006.
\end{abstract}

Keywords: multimodal biometrics, GLCM, Harlick feature, FAR, FRR

\section{Introduction}

There are number of applications where biometric technique are used to identify the person or claimant. As finger print, palm prints are mostly used as physiological traits, because of its characteristics such as distinctiveness, user friendly, low cost and so on. Therefore palm print finger print are the reliable methods for recognition. Many researchers have been proposed their algorithm for Biometric recognition methods for palm print recognition [2]-[4], [11] Fingerprint [5, 6, 7, 10] .Hand geometry [13].

The palm print based authentication system uses principle line, ridge ending, bifurcation point and textural information to form feature vector [2,5]. There are two palm print recognition techniques Statistical and structural [16].In first, methods are Eigen palm, Gabor filter, Fourier transform whereas gray level cooccurrence matrix, Harlick feature, textural based analysis are the statistical methods. Gabor filter is one of the technique to extract the feature of whole palm image or some region of palm, which is proposed by [2] and images are compared with hamming distance. A wavelet based textural information method is given by [4], Gabor phase encoding technique has been used in [3].

There are three different methods [1] of finger print recognition namely correlation based matching, minutiae based matching and non-minutiae based matching. Biometric system based on single biometric trait are known as unimodal system. Are usually cost effective solution as compared with multimodal system .However system using single biometric trait fail to be sufficient for authentication due to this multimodal system is useful which gives a better performance of system as compared with unimodal system.

A multimodal system based on Eigen palm and Eigen finger has been developed [20] in which matching score level fusion technique is implemented. In [22] fusion of iris and face biometric traits using weighted sum rule based matching score level fusion has been used. Our multimodal biometric recognition system based on texture feature extracted from palm images by means of GLCM based Harlick feature and minutiae based features of figure print .These features of finger and palm images are fused together at matching score level. Final decision of accepted or rejected is based on threshold value of matching score.

The rest of paper is arranged as follow. Section 2 gives proposed method for the fusion of palm and figure print biometric motilities .Section 3 and 4 palm print recognition and finger print recognition respectively. Matching score level is explained in section 5 .The experiment and results are discussed in section 6. The conclusion is given in section7.

\section{Proposed Recognition System}

In general, the proposed method consist of different steps as shown in Figure 1.Image processing has been done on palm and finger images using different techniques for each biometric trait. Then we extract the feature of palm print and finger print using different feature extraction techniques. 
Figure 1: Block diagram of Fusion of Palm and Finger Print



In the next stage matching score for each biometric trait dataset are obtained in the range of 0 to 1 .Next stage of the proposed system is fusion of palm print and finger print score has been done using weighted sum rule .In last stage that is after fusion of matching score, matching is decided if the fused score is greater than threshold value then user is genuine otherwise it is imposter.

\section{Palm Print Recognition System}

In the past few years palm print biometric authentication widely used methods for research. Many researcher and number of algorithm were developed and implemented for recognition. In our proposed research preprocessed segmented palm print images from IIT Delhi Touch less Palm print database is used. The most of information of textural feature exist in central part of palm therefore this part must be extracted from palm image which is known as Region of Interest (ROI). The segmented ROI palm images further processing of feature extraction.

\section{A. Gray level Co-occurrence matrix and Harlick Features:}

The one of the most useful statistical methods to the characterization of image texture is GLCM. Grey level co-occurrence matrix (GLCM) gives information about a distribution of intensities of a pixel and relative position of neighborhood pixel. This is used for gray level images [15]. GLCM can be calculated [14]. Suppose image to be analyzed is of rectangular and of size $n_{x}$ and $n_{y}$ columns and rows. Suppose $n_{g}$ is quantized gray level of a pixel, i.e., $\mathrm{Gx}=\left\{0,1,2, \ldots \ldots \ldots \mathrm{n}_{\mathrm{g}}\right\}$ are the set of $\mathrm{Mg}$ quantized gray level. Texture related information is defined by $\mathrm{Pij}$ value, is the probability value. From the GLCM that is how many time two neighboring pixels separated by distance $d$ with the grey level of reference pixel $i$ and a neighboring pixel with the grey level of $\mathrm{j}$. which gives a table of how often a different combination of pixel occur in an image to be analyzed. As we are interested in textural features, the following equations give these features. As Harlick proposed [14 ] fourteen textural features calculated from symmetrical normalized GLCM, we are using only following Harlick features in our scheme, are contrast, Autocorrelation, Energy, Homogeneity, Entropy.

GLCM are calculated for every sub image of ROI with different orientation $\left(0^{0}, 45^{\circ}, 90^{0}, 135^{\circ}\right)$ with considering eight and sixteen quantization level of gray values and distance between two neighboring pixel one .Then from this GLCMs Harlick feature are calculated of every sub images of palm print ROI. After that averaging of these Harlick features over all four orientation angle we get averaged value of all five Harlick feature for every sub images. Then by combining these values Harlick feature to single feature vector is formed for every ROI of palm print. Then we normalize this feature vector. In the matching, stage comparison is done between template and query (test) image. In this method, we use a Euclidean distance similarly method to decide the matching.

\section{Finger Print Recognition System}

The finger print recognition. One of the most reliable, oldest and mostly used secure biometric recognition is figure print. Finger print, preprocessing, training testing and matching are the different steps for figure print recognition process. For image preprocessing step method given in [21] has been used to detect ROI of figure print images. Here we are using FVC 2002 DB_B finger print data base. In selected ROI of finger print marking of minutiae points like ridge ending and bifurcation point has been done. The detected minutiae point is stored as a template for every finger image of training dataset.

DOI: $10.9790 / 1676-1203052731 \quad$ www.iosrjournals.org $28 \mid$ Page




\section{Fusion At Matching Score Level.}

One of the most important technique in our work is to build multimodal score vector of normalized palm print and figure print to verifies or recognize the claim of person genuine or imposter. This has been done by using machine score level fusion technique .In general there are four different technique of fusion of information in multimodal biometric system [1] Sensor level, Feature level, Machine score level and Decision Level

Out of these four method matching score level fusion is most popular method .As in this method different matching techniques provide score of different modalities in one range. Here by combining score of palm print and figure print to from individual scalar score to decide acceptance /rejection of claimant. There are different technique to combine this score based on product and weighted sum rule, weight can be decided by user specific or it can be calculated using equal error rate, distribution of scores, quality of individual biometric .In our scheme we are detecting the weight of individual modalities by accuracy of that particular unimodal system., Fused scores $\mathrm{S}$ is calculated with score of palm print (Ps) and finger print (Fs), given by following equation.

$\mathrm{S}=w 1 \mathrm{Sp}+w 2 \mathrm{Sf}$

Where $\mathrm{W} 1$ and $\mathrm{W} 2$ are weights if $\mathrm{a}_{1}$ is accuracy of palm print and $\mathrm{a}_{2}$ is accuracy of figure print then there weights are calculated as,

$w 1=\frac{a 1}{a 1+a 2} \quad$ and $\quad w 2=\frac{a 2}{a 1+a 2}$

Using this fused score the final decision is obtained in our proposed method.

\section{Experiments And Results}

The performance of Unimodal and multimodal system is evaluated on IIT-Delhi Database and FVC2002 DB_B database. We are assuming palm print and fingerprint of above dataset is from set of same person. Since palm print and fingerprint are independent from each other. In this study experiments are performed on IIT-Delhi Touch less palm print data set. Here we are chosen four images per person for training randomly from palm print dataset for ten users .Similarly we also randomly selected four finger print per person from FVC2002 Db21b dataset we also selects one image per unimodal system for testing.

\section{A. Experiment on Unimodal system.}

Finally experiments are carried out to measure the performance of palm print and finger print Unimodal system. We experimented our method on IIT Delhi Touch less palm print database Version1-0 [19]. Thus database mainly consists of hand images acquired by the Biometric research Laboratory using digital CMOS camera . These images are in bitmap format. This data base contains left and right-hand images of more than 230 subjects, at least five hand image samples from left as well as right hand. In addition to that, automatically segmented and normalized palm prints are available which are used in our method. The resolution of the images is $800 \times 600$ pixels with segmented cropped ROI images with size $150 \times 150$ pixels. For the evaluation of this algorithm, randomly selected 10 users are used with five images for each user. Out of this five user 04 images are used for template and 01 image per user for testing.

The ROI of palm print is the $150 \times 150$ pixel. Then we divide this ROI it into number of distinct sub images. The size of sub images should be at least greater than grey quantization level used for obtaining GLCM. As we are performing experimentation on 8 quantization level as well as 16 quantization level therefore we have chosen sub image size of $25 * 25$ pixels. When size of sub image is $25 * 25$ Pixels the ROI of palm print is divided into 36 sub images, we are extracting five Harlick feature namely contrast, Autocorrelation, Energy, Homogeneity, Entropy. It means we represent each ROI of palm print with $5 * 36=180$ features to form a feature vector. During comparison of query image again we follow same procedure to get feature vector of query image which is used in template formation. The matching score is decided on Euclidian distance to decide genuine and imposter claim. If value is greater than threshold then user accepted as genuine otherwise it is imposter. Experimentation results are given in following Table 1.

Table1. Experimental result for different quantization level with $25 * 25$ sub image size for palm print.

\begin{tabular}{|l|l|l|}
\hline Quantization level & Accuracy & EER \\
\hline 8 grey levels & $93.15 \%$ & 0.057 \\
\hline 16 grey levels & $91.74 \%$ & 0.059 \\
\hline
\end{tabular}

Similarly in finger print recognition minutiae based technique is used for recognition. We experimented our method on FVC 2002 DB2_B finger print database. Thus database mainly consists of finger images acquired by the optical sensor camera .These images are in bitmap format. This data base contains finger images of 10 subjects, with 8 images per subject. The resolution of the images is $296 \times 560$ pixels. For the evaluation of this algorithm, 10 users are used with five images for each user. Out of this five user 04 images are used for 
template and 01 image per user for testing. In this minutiae are represented and it is described as $\mathrm{Mi}=\{\mathrm{x}, \mathrm{y}, \theta\}$ where $\mathrm{x}, \mathrm{y}$, represents $\mathrm{x}, \mathrm{y}$ co-ordinates of the minutiae, $\theta$ is angular direction of the main ridge (minutiae angle).The main aim of minutiae based matching is to perform one to one mapping or paring of minutiae points from test data set minutiae of images .Let $\mathrm{D}$ and $\mathrm{T}$ are the query image and $\mathrm{T}$ stored template feature vector respectively.

$\mathrm{D}=\{\mathrm{m} 1, \mathrm{~m} 2, \mathrm{~m} 3, \ldots \ldots \ldots, \mathrm{mk}\} \quad \mathrm{mi}=\left\{\mathrm{x}_{\mathrm{i}} \mathrm{y}_{\mathrm{i}} \theta_{\mathrm{i}}\right\}, \mathrm{i}=1 \ldots \ldots \mathrm{K}$

$\mathrm{T}=\{\mathrm{m} 1, \mathrm{~m} 2, \mathrm{~m} 3, \ldots \ldots \ldots, \mathrm{mj}\} \quad \mathrm{mi}=\left\{\mathrm{x}_{\mathrm{j}} \mathrm{y}_{\mathrm{j}} \theta_{\mathrm{j}}\right\}, \mathrm{i}=1 \ldots \ldots \mathrm{L}$

Minutiae $m_{j}$ in the $T$ and in D minutiae are said to be matched if the spatial distance $\left(s_{d}\right)$ between them is smaller than $r_{0}$ and direction difference $(d d)$ smaller than angular tolerance $\left(\theta_{0}\right)$.given by following equations.

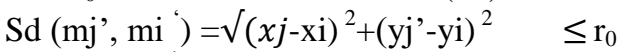

$\mathrm{dd}\left(\mathrm{mj}\right.$, mi $\left.{ }^{\prime}\right)=\min \left(\left|\theta_{\mathrm{j}},-\theta_{\mathrm{i} \mid}\right|, 360^{\circ}-\left|\theta_{\mathrm{j},-} \theta_{\mathrm{i} \mid}\right|\right) \leq \theta_{0}$

In order to maximize matching of minutiae alignment of stored and query template is used in most of application. Matching score can be found by calculating number of minutiae pair matched during matching of query image minutiae with each template stored in database. Matching score is calculated by taking ratio of number of minutiae matched against number of minutiae is the stored template. Table 2 gives accuracy and EER of finger print recognition system.

Table: 2 Experimental result for finger print recognition system.

\begin{tabular}{|l|l|l|}
\hline \multirow{2}{*}{ Finger print } & Accuracy & EER \\
\cline { 2 - 3 } & $97.53 \%$ & 0.024 \\
\hline
\end{tabular}

\section{B. Experiment on Multimodal System:}

Fusion of palm print and finger print score gives high recognition accuracy as compared with individual Unimodal biometric system alone. Here Fusion of matching score is achieved using weighted sum rule. The result or the Fusion of two traits i.e. palm print and finger print gives better performance compared to Unimodal system. The weighted sum rule based Fusion gives better accuracy. This result is given in the Table 3 .

Table3: Experimental result palm print, finger print and fusion of palm, finger print.

\begin{tabular}{|l|l|l|}
\hline Biometric trait & Accuracy & EER \\
\hline Palm print & $93.15 \%$ & 0.057 \\
\hline Finger print & $91.74 \%$ & 0.059 \\
\hline Palm + Finger Print & $99.93 \%$ & 0.0006 \\
\hline
\end{tabular}

Our proposed palm-finger print Multimodal system is compared with Unimodal systems ROC (Receiver Operator Characteristics) analysis. Threshold values will decide the accuracy as well as FAR, FRR values. For different threshold we will get different values of FAR, FRR. The probability of FAR versus FRR is plotted for different values of decision threshold as shown in Figure 2. In Figure 3 along with EER (Equal Error Rate) given on set top of the curves. Where values of FRR and FAR are equal. The ERR of palm print Finger print respectively. Whereas ERR of Multimodal system from ROC curve where improvement of performance of Multimodal system over Unimodal system is seen in Figure 3.

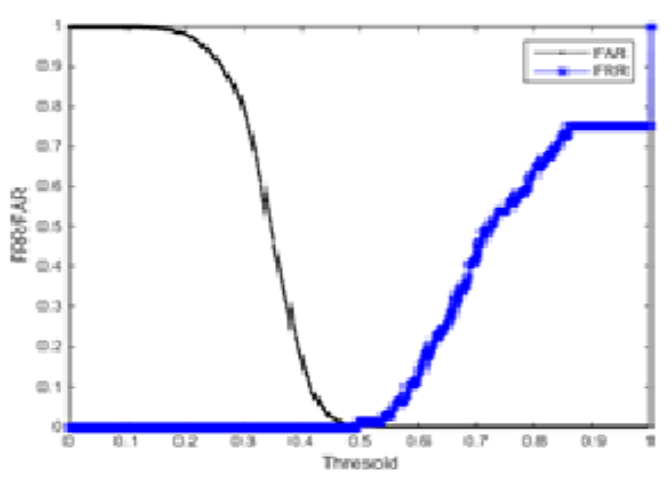

Figure 2: Threshold verses FAR/FRR

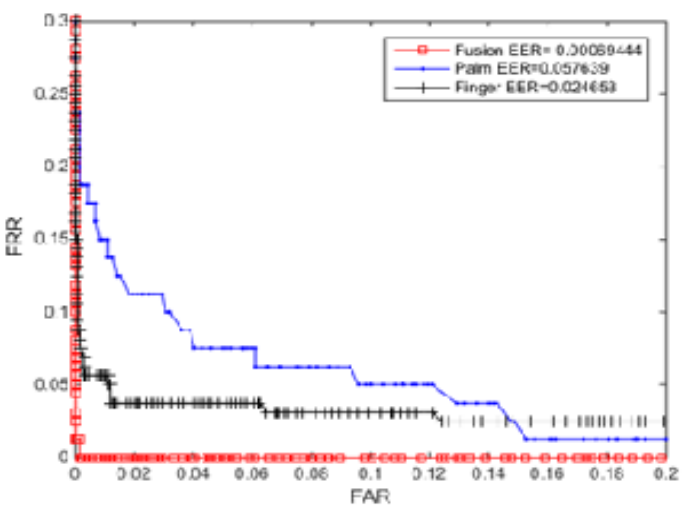

Figure 3: ROC Curve

\section{Conclusion}

Fusion of palm print and finger print biometrics using different feature extraction, feature normalization and weighted sum based rule is used to evaluate performance of our proposed multimodal system. In palm print recognition system GLCM based Harlick features are extracted to form feature vector where as in finger print minutiae based method has been used to form feature vector. The experimentation has 
been carried out on virtual multimodal database. The comparative experiments ascertain that the proposed weighted sum fusion approach outperforms the other sum fusion approaches. The experimental results show that our fusion of Fingerprint and palm print performs well.

\section{References}

[1]. A.K. Jain, A. Ross, S. Prabhakar "An introduction to biometric recognition", IEEE Trans .circuits Systems, video 14(1) (special issue on image and video based biometrics pp 4-20, 2004.

[2]. A. Kong and D. Zhang "Palm texture analysis base on low-resolution images for personal authentication." In the processing of 16 th International conference on Pattern Recognition, Vol. 3. PP 807-810, 2002.

[3]. D. Zhang, W. K. Wang. J You and M. Wong, "Online Palm print identification" IEEE Transactions on Pattern Analysis and Machine Intelligence. Vol.25 no. 3- 1041-1050.

[4]. Mustafa Mumtaz et. al. "Wavelet-based palm print Authentication system" Biometrics and security Technology, 2008, International symposium on 23-24 April 2008.

[5]. X.Wu, D Zhang, K. Wang and B. Huang. "Palm print classification using Principal lines" Pattern Recognition letters, Vol 24, no.10, pp 1987-1998, 2004.

[6]. G. Lu, D Zhang, K. Wang. "Palm print recognition using Eigen palms features" Pattern Recognition letters, Vol 24, no.09, pp 14631467, 2003.

[7]. Mushi p.ry.al. "A rough set based binarization techniques for fingerprint images" Signal processing, computing and control (ISPCC)2012.IEEE International conference on 15-17 march 2012.

[8]. chin-Chuan Han, Hsu - Liang chengm Ching Lun Ling, Kuo-chin fan, "Personal authentication using Palm print features" Pattern Recognition 36(2003) 371-381.

[9]. V.V. Satyanarayana, Tallapragada, E. ., Rajan, "Iris recognition based on combined feature of GLCM and wavelet Transform" First International conference on Integrated Intelligent Computing” PP-205-210.

[10]. Shashikumar D.R. et.al. "DWT based fingerprint Recognition using Non-Minutiae features" International journal of computer Science, issues 02 March 2011.

[11]. Ali Younesi and Methi chehel Amirani. "Palm print identification via GLCM of Contourlet Transform" 978-1-4673-2821.

[12]. Tee Connie Andrew Tech Beng Jin, Michel Goh-Koh Ong, David Ngo check Ling, "An automated palm print recognition system" Image and Vision computing 23(2005) PP 501-515.

[13]. Goh Koh Ong Michel, Tee Connie, Andrew Tech, David NGO, “Automated Hand geometry verification system bases on Silent points."

[14]. Robert M. Harlick, K. Shanmugam and ITS'Hak Dinstein. "Textural feature for image classification" IEEE Transaction on systems Man and Cybernetics. Vol- SMS No. 6 November 1973 pp610-621.

[15]. Li Liu, Longfei Yang, Yizheng Wang, Aigi Yang "Combining Gray-level co-occurrence Matrix and statics feature for Rotation Invariant Texture classification in Wavelet Domain" 8th International congress on Image and Signal Processing (CISP-2015) pp539-543.

[16]. Y.L. Malathi Latha and Munaga V. N. Prasad. "GLCM based Texture features for palm print identification system" Computational Intelligence in Data Mining -Vol-1, smart Innovation system, and Technologies, 31.

[17]. Aditya Nigam Phalguni Gupta," Designing and accurate hand biometric-based authentication system fusing finger khukleprint and palm print" Neurocomputing 151(2015) pp-1120-1132.

[18]. Medha Misar, Damayanti Gharpure "Extraction of feature vector based on wavelet coefficient for palm print based Biometric Identification system." 2nd International symposium on physics and technology of sensors, 8-10 March 2015. Pp-113-119,

[19]. https"//web.iitd.ac.in/ ajaykar/database-palm.htm - "IIT Delhi Touch less Palm print database version 1.0"

[20]. Slobodan Ribaric, Member, IEEE, and Ivan Fratric "A Biometric Identification System Based on Eigen palm and Eigen finger Features “,IEEE TRANSACTIONS ON PATTERN ANALYSIS AND MACHINE INTELLIGENCE, VOL. 27, NO. 11, NOVEMBER 2005

[21]. Hong, L., Wan, Y. \& Jain, A. (1998).” Fingerprint image enhancement: Algorithm and performance evaluation, "IEEE Transactions on Pattern Analysis and Machine Intelligence" 20(8): 777-789.

[22]. Maryam Eskandari - Önsen Toygar" Fusion of face and iris biometrics using local and global feature extraction methods" SIViP December 2012 